\title{
The Study Of The Arch Types Research And Prevention Measures About The Grain Inside Silos
}

\author{
Yong Feng ${ }^{1, a^{*}}$,Feiya Xie ${ }^{1, b}$ \\ ${ }^{1}$ Henan University of Technology, Henan Zhengzhou China,450000 \\ a51433719@qq.com,'932478698@qq.com
}

Keywords: slio;mechanism; struct types; prevention

Abstract. An arch will block the flow of grain, destroy the continuity and reliability of the discharge operations, and interrupt the continuity of discharging. Aim at the arch could produce harmful phenomenon and combining with the knot into the mechanism of the arch, when we load and unload food.We propose the arch type of silo, and take effective prevention to control the arch.

\section{Introduction}

The granary is the main way to store grain, when we discharge the grain,due to water being big,wetness being high, storage time being too long, and materials with the warehouse wall adhesion, the structure of the bunker, the structure of overside port and the angle of hopper, whith can lead to an arch.The study about arch can be dated back to the resarch of mechanics of granular media ,in history.Bulk material has much diffent machanical property from solid and liquid. So a lot of people make a study on this for a long time.

\section{The silos within the grain of arch type}

According to different causes of the formation of the arch ,there are four types about the arch,Compression arch, Cuneatic arch,Adhesion consolidation arch,and Pressure balance arch.They are shown in Fig.1.

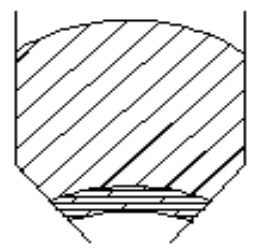

Compression arch

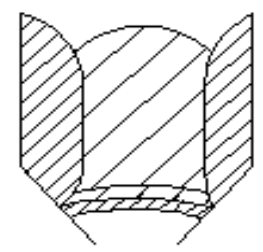

Adhesion consolidation arch

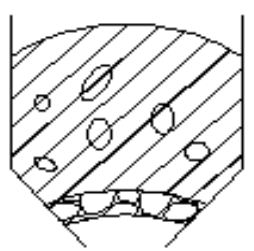

Cuneatic arch

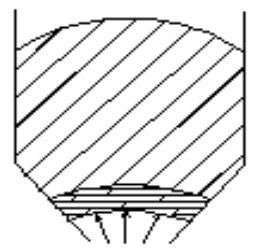

Pressure balance arch

Fig.1. Arch types

The Compression arch. This tyope of arch fomation is due to the grain being pressed by the materrial of itself,the pressure inside the silo, or material volume expansion after squeezing each other., which increase the concretion strength of grain and form the compression arch.

The Cuneatic arch. When the grian unloading ,the irregular of the grain can cause interlocking between particles, which makes the interparticle force banlances each other.That will make grain unloaded confusing and form wedge arch at discharging port. 
Adhesion consolidation arch. The humidity foodstuff particle itself, viscosity, the action of gravity,and the influence of the performance of the warehouse wall material, which increas adhesive with the warehouse wall that lead to adhesion consolidation arch, when the grain in unloaded.

Pressure balance arch. The silo air tightness is poor, which results a large number of air into the silo bottom and the fluctuation pressure being balanced, which will form the pressure balance arch.

\section{The mechanism of arch of the food within the silos}

There are many reasons for the formation of grain in the silos.Such as ,the shape of the silo,physical properties of food and the grain moisture.

Whether there will be formed arch phenomenon, and it related to the shape of silo.Japan's wood long one puts forward that there is a critical size control for the formation of the arch.

The computation formula is:

$$
D_{C}=\frac{4 \mathrm{c}}{r}(1+\sin \varphi \mathrm{i})
$$

$D_{C} \longrightarrow$ The size of the material and arch export

$\mathrm{C}$ - The pressure of materials and the storage time correlation coefficient

$\mathrm{R}$ — Material density ;

$\varphi \mathrm{i} \_$Angle of internal friction material

The capsule structure affects the food grain surface shear strength size to a certain degree,So thebin structure parameter affects the liquidity of the material.

At the end of the 17th century,Cullen is "packing pressure on retaining wall "studied and put forward the coulomb's law:

$$
\tau=\sigma \operatorname{tg} \varphi_{i}+c
$$

$\tau \_$shear stress $\sigma$ Shear plane normal stress

c___binding power $\varphi_{\mathrm{i}}$ Angle of internal friction material

After many years of study,Yang sen concluded that deep warehouse material of wall and warehouse bottom pressure equation, in 1895 .

$$
\begin{aligned}
& \frac{W R}{\mu}[1-\exp (-K \mu h / R)] \\
& \varepsilon=W R\left\{k-\frac{R}{K \mu}[1-\exp (-K \mu h)]\right\}
\end{aligned}
$$

L__idepressure $\quad \mathrm{W}-$ __olume density

$\mathrm{R}-$ Radius hydraulic $\mathrm{h}-$ Calculate the material deep place

$\mu-$ Coefficient of friction $\mathrm{K}-$ side pressure coefficient

$\varepsilon-$ vertical pressure

In the 1960s,Enike, Johanson and Walters made a lot of research and established the basic theory of material flow in the bin. That is shown in Fig.2.

As bin material is not the whole flow of the pressure distribution in the following. We can put forward the theory: 
If $\sigma_{\mathrm{c}}<\overline{\sigma_{1}}$ The material flow as a whole

If $\sigma_{\mathrm{c}}=\overline{\sigma_{1}}$ The material will knot arch

If $\sigma_{c}>\overline{\sigma_{1}}$ The material flow occlusion is not

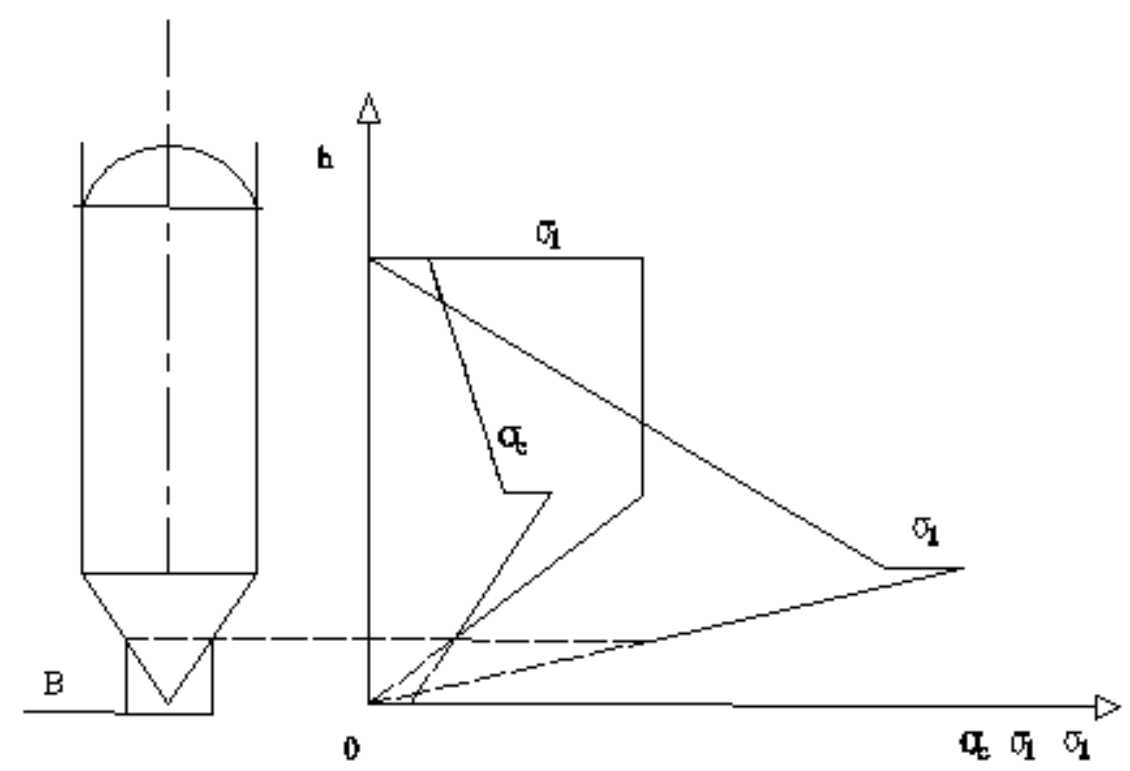

Fig. 2. Stress analysis of the overall flow of bunker

$\sigma_{C}$ - The intensity of material surface $\sigma_{1}$ - Material compressive stress $\overline{\sigma_{1}}-$ Material axial stress

$\mathrm{h}$ - deep of the material B - Bin exit diameter

The research perspective can be summed up,the formation of the arch is relsted to the food itself physical properties(Moisture conten,Angle of internal friction material), the structure of the bin and the food and the adhesion of food with the silo wall.

\section{The measures to prevent silo, grain knot arch}

Because silo granary of arch affects the orderly conduct of social production,we need according to the principle of silo and the grain of arch theory,putting forward effective inhibition arch measure.

Strictly control the grain moisture. We know the moisture content of grain and the soil content of grain will increase food caking,and studies have shown that we must strictly control the grain moisture,we should make the water shall not exceed $15 \%$. So food before entering the silo should be conducted drying process.

To optimize the design of the silo. Slio has two parts :the upper barrel and the cone in the bottom. And the gradient of the cone in the bottom is an important factor for the arch. Regarding the silo in which the materil is buik grain,experiments show that when we design the angle, it should be greater than 45 degrees. At the same time, when design the discharge holewe, satisfying bottom storehouse slope angle,we should choose rectangle.The reason is square discharging mouth is difficult to form an arch,compared with round discharging mouth.

\section{Adding auxiliary device}

Adding electric vibrator. The working principle of the electric vibrator is useing vibrator to shake warehouse wall, and the effects of the vibration of material will prevent and ruled out the arch .Dueing the friction material, charged, deliquescence, composition segregation silo block to developing arch. The way is a relatively simple method of arch breaker. When we use it, the frequency of the vibrator shuld be strictly controled for reducing damage to the silo. 
Increase pneumatic broken GongQi device. The arch breaker uses strong airflow produced by compressed air,and directly thrust forth into the storage medium material block fault zone at supersonic speeds.The sudden release of the expansion of the shock wave can overcome the static friction material,and make the container of the material flow back again. The arch breaker has many advantages, for instance,simple structure, convenient use safety, huge impact, security, energy saving, automatic control, simple operation, no damage to warehouse and so on.It is the most ideal arch breakerand flow device.

Add a silo internal auxiliary device. We can set up some baffle inside the silo .The baffle can make food for shunt, and improve its liquidity aslo, the baffle can ease the pressure on grain exports to bin when outflow.There are some common types of damper,for example,Horizontal baffle(Fig3a),Verticalbaffle(Fig3b),Slopingbaffle(Fig3c),Invertedcone(Fig3d),Bipyrmid(3e).They are shown in Fig.3.

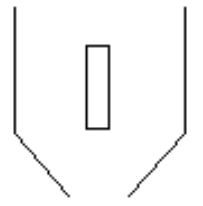

由

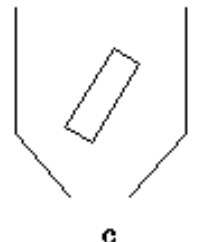

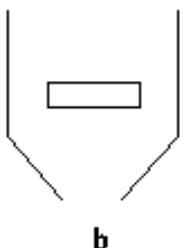

b

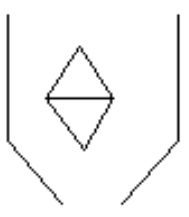

$\theta$

Fig.3. Baffle types

\section{Improve the material silo}

When storage grain, we should use smooth surface of the steel plate as far as possible to make grain elevator.If conditions allowed, can we add a layer of plastic in the steel plate surface to ensure warehouse wall and warehouse bottom are smooth.If the material selection of silo is the concrete material,we should do smooth processing to the warehouse wall and warehouse at the end of the inner surface.That can reduce the friction silos for food, and increase their liquidity.

\section{Conclusion}

Through effective prevention measures, can we avoid some extent the silos within grain and the formation of the arch. We should according to the actual situation of different,and adopt high efficiency and energy saving ways to deal with the arch of grain. Only in this way, can we ensure the production continues. Although we have some arch prevention measures and broken arch ways, we need to work harder for the arch of the in-depth study.

\section{Acknowledgements}

This work was financially supported by the 2014 special food public welfare research project "research and development of new type storehouse and technical system of ecological grain storage " and 2016 key scientific research project for colleges and universities in henan province . 


\section{References}

[1]Walkters J R. A Thearetical Analysis of Stresses in Axially Symmetric Hoppers and Bukers Chem.Engng . Sci. 1973,(28) 779.

[2]Jenike.AW.GravityFlowofBulkSohd.Bul.108.UtahEngng.Exper.Scacion.Universityof Utah.1962.

[3]JewellRA, Wroth CP . Direct shear tests On reinforced sand[J] . Geotechique, 1987,37(1) : $53-68$.

[4] W. Guan, Q. Zhang . The effect of moisture content and compaction on the strength and arch formation of wheat flour in a model bin. Journal of Food Engineering 94 (2009) 227-232. 